\title{
Clinical characteristics and outcomes of inpatients with neurologic disease and COVID-19 in Brescia, Lombardy, Italy
}

\begin{abstract}
Alberto Benussi, MD, Andrea Pilotto, MD, Enrico Premi, MD, llenia Libri, MD, Marcello Giunta, MD, Chiara Agosti, MD, Antonella Alberici, MD, Enrico Baldelli, MD, Matteo Benini, MD, Sonia Bonacina, MD, Laura Brambilla, MD, Salvatore Caratozzolo, MD, Matteo Cortinovis, MD, Angelo Costa, MD, Stefano Cotti Piccinelli, MD, Elisabetta Cottini, MD, Viviana Cristillo, MD, Ilenia Delrio, MD, Massimiliano Filosto, MD, Massimo Gamba, MD, Stefano Gazzina, MD, Nicola Gilberti, MD, Stefano Gipponi, MD, Alberto Imarisio, MD, Paolo Invernizzi, MD, Ugo Leggio, MD, Matilde Leonardi, MD, Paolo Liberini, MD, Martina Locatelli, MD, Stefano Masciocchi, MD, Loris Poli, MD, Renata Rao, MD, Barbara Risi, MD, Luca Rozzini, MD, Andrea Scalvini, MD, Francesca Schiano di Cola, MD, Raffaella Spezi, MD, Veronica Vergani, MD, Irene Volonghi, MD, Nicola Zoppi, MD, Barbara Borroni, MD, Mauro Magoni, MD, Alessandro Pezzini, MD, and Alessandro Padovani, MD, PhD
\end{abstract}

Neurology ${ }^{\circledR}$ 2020;95:e910-e920. doi:10.1212/WNL.0000000000009848

\section{Correspondence} Dr. Padovani alessandro.padovani@ unibs.it
MORE ONLINE

\section{COVID-19 Resources}

For the latest articles, invited commentaries, and blogs from physicians around the world NPub.org/COVID19

\section{Methods}

In this retrospective, single-center cohort study, we included all adult inpatients with confirmed COVID-19 admitted to a neuro-COVID unit beginning February 21, 2020, who had been discharged or died by April 5, 2020. Demographic, clinical, treatment, and laboratory data were extracted from medical records and compared (false discovery rate corrected) to those of neurologic patients without COVID-19 admitted in the same period.

\section{Results}

One hundred seventy-three patients were included in this study, of whom 56 were positive and 117 were negative for COVID-19. Patients with COVID-19 were older (77.0 years, interquartile range [IQR] 67.0-83.8 years vs 70.1 years, IQR 52.9-78.6 years, $p=0.006)$, had a different distribution regarding admission diagnoses, including cerebrovascular disorders $(n=43,76.8 \%$ vs $\mathrm{n}=68,58.1 \%$ ), and had a higher quick Sequential Organ Failure Assessment (qSOFA) score on admission (0.9, IQR $0.7-1.1$ vs 0.5 , IQR $0.4-0.6, p=0.006)$. In-hospital mortality rates $(\mathrm{n}=21$, $37.5 \%$ vs $\mathrm{n}=5,4.3 \%, p<0.001)$ and incident delirium $(\mathrm{n}=15,26.8 \%$ vs $\mathrm{n}=9,7.7 \%, p=0.003)$ were significantly higher in the COVID-19 group. Patients with COVID-19 and without COVID with stroke had similar baseline characteristics, but patients with COVID-19 had higher modified Rankin Scale scores at discharge (5.0, IQR 2.0-6.0 vs 2.0, IQR 1.0-3.0, $p<0.001$ ), with a significantly lower number of patients with a good outcome $(n=11,25.6 \%$ vs $n=48,70.6 \%, p<$ 0.001). In patients with COVID-19, multivariable regressions showed increasing odds of inhospital death associated with higher qSOFA scores (odds ratio [OR] 4.47, 95\% confidence interval [CI $] 1.21-16.5, p=0.025$ ), lower platelet count (OR 0.98, 95\% CI 0.97-0.99, $p=0.005$ ), and higher lactate dehydrogenase (OR 1.01, 95\% CI 1.00-1.03, $p=0.009$ ) on admission.

\section{Conclusions}

Patients with COVID-19 admitted with neurologic disease, including stroke, have a significantly higher in-hospital mortality and incident delirium and higher disability than patients without COVID-19.

From the Neurology Unit (A.B., A. Pilotto, I.L., M.G., E.B., S.B., M.C., S.C.P., V.C., A.I., M. Locatelli, S.M., B.R., L.R., A.S., F.S.d.C., N.Z., B.B., A. Pezzini, A. Padovani), Department of Clinical and Experimental Sciences, University of Brescia; Neurology Unit (A.B., A. Pilotto, C.A., A.A., S.C., E.C., M.F., S. Gipponi, P.L., L.P., R.R., L.R., I.V., B.B., A. Pezzini, A. Padovani), Vascular Neurology Unit (E.P., A.C., I.D., M.G., N.G., R.S., V.V., M.M.), and Neurophysiology Unit (S. Gazzina, U.L.), Department of Neurological and Vision Sciences, ASST Spedali Civili, Brescia; Neurology Unit (M.B.), University of Bologna; Department of Neuroimmunology and Neuromuscular Diseases (L.B.) and Neurology (M. Leonardi), Public Health and Disability Unit, Foundation IRCCS Neurological Institute Carlo Besta, Milan; and Neurology Unit (P.I.), Fondazione Poliambulanza Hospital, Brescia, Italy.

Go to Neurology.org/N for full disclosures. Funding information and disclosures deemed relevant by the authors, if any, are provided at the end of the article. 


\section{Glossary}

$\mathbf{C I}=$ confidence interval; COVID-19 = coronavirus disease 2019; IQR = interquartile range; $\mathbf{m R S}=$ modified Rankin Scale; NIHSS = NIH Stroke Scale; qSOFA = quick SOFA; SARS-CoV-2 = severe acute respiratory syndrome coronavirus 2; SOFA = Sequential Organ Failure Assessment.

Since February 20, 2020, Lombardy, Italy, has experienced a major outbreak of coronavirus disease 2019 (COVID-19), caused by the severe acute respiratory syndrome coronavirus 2 (SARS-CoV-2), with $>50,000$ cases and 9,000 deaths in the region as of April 5, 2020.

The clinical spectrum of SARS-CoV-2 appears to be wide, encompassing asymptomatic infections, mild upper respiratory tract illness, and severe pneumonia with respiratory failure and death. ${ }^{2}$ Several factors have been associated with increased mortality, including older age, high Sequential Organ Failure Assessment (SOFA) score, and increased d-dimer levels. ${ }^{3}$

To date, only 2 retrospective case series from convenience samples from 3 hospitals in Wuhan, China, have been published ${ }^{4}$ or posted on preprint servers without peer review. ${ }^{5}$ The most common neurologic manifestations were dizziness (16.8\%), headache (13.1\%), and encephalopathy (2.8\%). Stroke complicated COVID-19 infection in 5.9\% of cases, with patients being older with more cardiovascular comorbid conditions and more severe pneumonia.,

It is still unclear whether patients with neurologic disease and COVID-19 have a different neurologic outcome compared to patients without COVID-19 and if this is achieved at the cost of days of hospitalization or increased mortality. Moreover, it is not known if stroke severity at admission and stroke severity at discharge are similar in the 2 populations and if acute-phase treatments such as endovascular therapy and IV fibrinolysis have similar outcomes. We aimed to describe the clinical and laboratory characteristics, treatment, and clinical outcomes of patients with neurologic disease with and without COVID-19.

\section{Methods}

\section{Standard protocol approvals, registrations, and patient consents}

This study received approval from the ethics standards committee on human experimentation (local ethics committee of the ASST Spedali Civili Hospital, Brescia: NP 4051, approved April 6, 2020). The requirement for informed consent was waived by the ethics commission for patients who were no longer alive or reachable at the time of approval, while full written informed consent was required for all other participants.

\section{Study design and participants}

This retrospective cohort study included adult inpatients $(\geq 18$ years old) admitted primarily for neurologic disease from the General Neurology Unit and Vascular Neurology Unit, Department of Neurologic and Vision Sciences, ASST Spedali Civili Hospital, Brescia, Italy, from February 21 to April 5, 2020. This hospital in Brescia was designated as a hub for acute cerebrovascular diseases during the COVID-19 outbreak in a metropolitan area of $>1,200,000$ people. ${ }^{7}$ The original units were divided into 2 separate sections for patients affected (neurological neuro-COVID unit) and not affected (non-COVID unit) by COVID-19, and staff neurologists were equally divided between the 2 units. ${ }^{8}$

Our study included all adult inpatients who were hospitalized for neurologic diseases and had a definite outcome (discharge home or to a rehabilitation facility or death).

The criteria for discharge for patients with COVID-19 were absence of fever for at least 24 hours, a respiratory rate $<22$ breaths/min, and substantial improvement at chest $\mathrm{x}$-ray or CT scan.

\section{Data collection}

Epidemiologic, demographic, clinical, laboratory, treatment, and outcome data were extracted from both printed and electronic medical records with standardized anonymized data collection forms. All data were imputed and checked by 4 physicians (A.B., A. Pilotto., M.G., and I.L.). The admission data of included patients ranged from February 21 to April 5, 2020.

\section{Demographical and clinical data}

The following demographic and clinical data were acquired for all patients, which were present on admission: age, sex, smoking habits, comorbid conditions (diabetes mellitus, hypercholesterolemia, hypertension, coronary heart disease, malignancy, chronic kidney disease, immunodeficiency), quick SOFA (qSOFA) score, premorbid modified Rankin Scale (mRS) score, and NIH Stroke Scale (NIHSS) score (for cerebrovascular disease only); during hospitalization: antibiotic, antiviral, or high-flow oxygen therapy, in-hospital mortality, incident delirium, fever during hospitalization, number of diagnostic tests, acutephase therapies such as IV fibrinolysis, endovascular therapy, or bridging therapy (IV fibrinolysis followed by endovascular therapy) (for ischemic stroke only); or at discharge: days of hospitalization, mRS score, and NIHSS score (for cerebrovascular disease only). The qSOFA score uses 3 criteria, assigning 1 point for low systolic blood pressure $(\leq 100 \mathrm{~mm} \mathrm{Hg})$, high respiratory rate $(\geq 22$ breaths/min), or altered mentation (Glasgow Coma Scale score $<15$ ), with a range from 0 (least impairment) to 3 (greatest impairment). 


\section{Laboratory procedures}

SARS-CoV-2 detection in respiratory specimens was performed by real-time reverse-transcriptase PCR methods, as described elsewhere. ${ }^{9}$ Both nasopharyngeal and oropharyngeal swabs were performed in all patients. If 2 consecutive tests obtained at least 24 hours apart were negative and there was high suspicion of COVID-19 (i.e., interstitial pneumonia at chest x-ray, low arterial partial pressure of oxygen), a bronchoalveolar lavage was performed.

Routine blood examinations comprised complete blood count, erythrocyte sedimentation rate, serum biochemical tests including C-reactive protein, liver and renal function, lactate dehydrogenase, creatine kinase, high-sensitivity troponin $\mathrm{T}$, serum ferritin, and coagulation profile. Highsensitivity troponin $\mathrm{T}$, ferritin, and d-dimer were performed in only a subset of patients $(\approx 20 \%)$.

\section{Definitions}

Fever was defined as axillary temperature of at least $37.5^{\circ} \mathrm{C}$. The diagnosis of delirium was defined by the presence of features 1 and 2 and either 3 or 4 of the Confusion Assessment Method. ${ }^{10}$ Antiviral treatment was defined as lopinavir/ritonavir 400/ $100 \mathrm{mg}$ twice daily, darunavir $800 \mathrm{mg}$ once daily + ritonavir $100 \mathrm{mg}$ once daily, or darunavir/cobicistat $800 / 150 \mathrm{mg}$ once daily. In patients with stroke, a good outcome was defined as an mRS score $\leq 2$. Diagnostics tests were defined as MRI (head), CT (head/thorax/abdomen), x-ray (thorax/abdomen), EEG, echography (heart/neck), and Holter monitor.

\section{Statistical analysis}

Continuous and categorical variables are reported as median (interquartile range $[\mathrm{IQR}]$ ) and number (percent), respectively. Differences between patients with and without COVID-19 were compared by the Mann-Whitney $U$ test, $\chi^{2}$ test, or Fisher exact test as appropriate.

To explore the risk factors associated with in-hospital death, univariable and multivariable logistic regression models were implemented. For multivariate analysis, to avoid overfitting in the model, variables were chosen on the basis of previous findings and clinical constraints. ${ }^{3}$ Previous studies have shown age, qSOFA scores, and several laboratory findings to be associated with in-hospital mortality. Therefore, we chose age, qSOFA scores, platelet count, C-reactive protein, and lactate dehydrogenase for our multivariable logistic regression model.

A 2-sided value of $p<0.05$ was considered significant and corrected for multiple comparisons with the Benjamini-Hochberg false discovery rate. ${ }^{11}$ Data analyses were carried out with SPSS software (version 21.0; Armonk, NY) and GraphPad Prism (version 8.0; GraphPad Software, La Jolla, CA).

\section{Data availability}

All study data, including raw and analyzed data, and materials will be available from the corresponding author on reasonable request.

\section{Results}

Two hundred fourteen adult patients were hospitalized in the Neurology and Vascular Neurology Unit of the ASST Spedali Civili di Brescia Hospital from February 21 to April 5, 2020. After the exclusion of 41 patients who were still hospitalized as of April 5, 2020, we included 173 inpatients in the final analysis. Of these, 56 (32.4\%) resulted positive for COVID-19 and were admitted to the neuro-COVID unit (figure).

Demographic, clinical, and laboratory characteristics of included patients are reported in table 1 (between-group differences with $95 \%$ confidence intervals [CIs] are reported in table e-1, links.lww.com/WNL/B124).

Results are reported as median (IQR) or number (percent), while false discovery rate-adjusted $p$ values for multiple comparisons are reported for each test. Compared to patients without COVID-19, patients with COVID-19 were older (77.0 years, IQR 67.0-83.8 years vs 70.1 years, IQR 52.9-78.6 years, $p=0.006$ ), had a different distribution regarding admission diagnoses, particularly for cerebrovascular disorders $(n=43$, $76.8 \%$ vs $\mathrm{n}=68,58.1 \%, p=0.035$ ), and had a higher qSOFA score on admission (0.9, IQR $0.7-1.1$ vs 0.5 , IQR $0.4-0.6$, $p=0.006)$. No significant differences were observed for comorbid conditions, including diabetes mellitus, hypercholesterolemia, hypertension, coronary heart disease, chronic kidney disease, immunodeficiency, or malignancy (all $p>$ 0.050). Patients with COVID-19 had higher in-hospital mortality ( $\mathrm{n}=21,37.5 \%$ vs $\mathrm{n}=5,4.3 \%, p<0.001)$ and higher incidence of delirium $(\mathrm{n}=15,26.8 \%$ vs $\mathrm{n}=9,7.7 \%, p=0.003)$ and fever $(\mathrm{n}=27,48.2 \%$ vs $\mathrm{n}=14,12.0 \%, p<0.001)$, while days of hospitalization were similar ( $\mathrm{n}=6.0 \mathrm{IQR} 3.3-10.0 \mathrm{vs}$ 5.0, IQR 4.0-8.0, $p=0.424$ ). Of patients who were discharged (excluding in-hospital deaths), days of hospitalization were increased in patients with COVID-19 (8.0, IQR 5.0-11.0 vs 5.0, IQR 4.0-8.0, $p=0.005$ ).

Treatments were different between the 2 groups, with a wider use of high-flow oxygenation $(n=43,76.8 \%$ vs $n=11,9.4 \%, p$ $<0.001)$, antibiotic therapy $(\mathrm{n}=36,67.9 \%$ vs $\mathrm{n}=19,16.2 \%, p$ $<0.001)$, and antiviral treatments $(\mathrm{n}=38,67.9 \%$ vs $\mathrm{n}=2$, $1.7 \%, p<0.001$ ) in the COVID-19 group.

Laboratory analysis showed an increased neutrophil and platelet count; reduced lymphocyte count; and increased C-reactive protein, erythrocyte sedimentation rate, highsensitivity troponin $\mathrm{T}$, lactate dehydrogenase, aspartate and alanine aminotransferase, prothrombin time, and fibrinogen (all $p<0.05$ ) in patients with COVID-19 compared to patients without COVID-19. No differences were observed for whole white blood cell count, hemoglobin, bilirubin, creatine kinase, creatinine, activated thromboplastin time, d-dimer, or serum ferritin (all $p>0.05)$ (table 1$)$.

Patients with COVID-19 had worse functional outcomes as measured by the mRS (5.0, IQR 2.3-6.0 vs 2.0, IQR 1.0-3.0, 


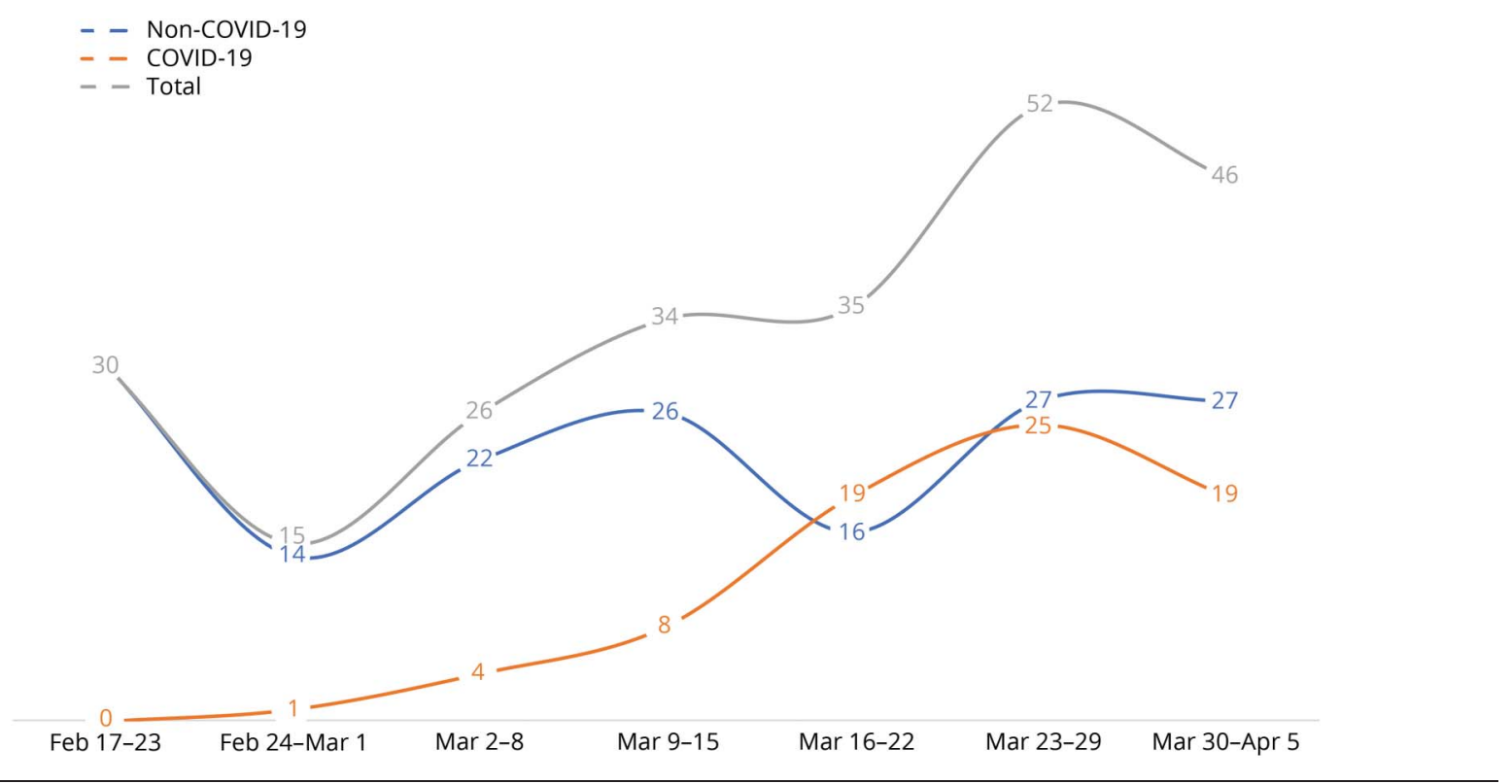

$p<0.001$ ), with similar premorbid mRS scores $(1.0, \mathrm{IQR}$ $1.0-2.0$ vs 1.0 , IQR $0.0-2.0, p=0.903$ ).

We observed a significant increase in cerebrovascular disease rates in the COVID-19 group ( $\mathrm{n}=43,76.8 \%$ vs $\mathrm{n}=68,58.1 \%$, $p=0.018)$, with a similar distribution among TIA $(\mathrm{n}=5$, $11.6 \%$ vs $n=8,11.9 \%)$, ischemic stroke $(n=35,81.4 \%$ vs $n=$ $50,74.6 \%)$, and hemorrhagic stroke $(\mathrm{n}=3,7.0 \%$ vs $\mathrm{n}=9$, $13.4 \%$ ) within groups, $p=0.560$ (table 2; between-group differences with 95\% CIs are reported in table e-2, links.lww. $\mathrm{com} / \mathrm{WNL} / \mathrm{B} 124)$.

Patients admitted for ischemic stroke had similar baseline characteristics, including sex, comorbid conditions, premorbid mRS score, and NIHSS score on admission. There were no differences in access to acute-phase therapies such as endovascular treatment $(\mathrm{n}=2,5.0 \%$ vs $\mathrm{n}=8,13.8 \%, p=$ $0.785)$, IV fibrinolysis ( $\mathrm{n}=4,10.0 \%$ vs $\mathrm{n}=2,3.4 \%, p=0.384$ ), or bridging therapy ( $\mathrm{n}=3,8.6 \%$ vs $\mathrm{n}=3,6.0 \%, p=0.785)$. Patients with COVID-19 had higher mRS scores at discharge (5.0, IQR 2.0-6.0 vs 2.0, IQR 1.0-3.0, $p<0.001$ ), with a significantly lower number of patients with a good outcome ( $\mathrm{n}=$ $11,25.6 \%$ vs $\mathrm{n}=48,70.6 \%, p<0.001$ ). This difference in outcomes was also confirmed considering only patients who underwent acute-phase therapies (mRS score at discharge $p=$ 0.009, good outcome $p=0.047$ ).

Moreover, patients with cerebrovascular disease (including TIA and ischemic and hemorrhagic stroke) and COVID-19 had an increased platelet count, reduced lymphocyte count, and higher $\mathrm{C}$-reactive protein, erythrocyte sedimentation rate, lactate dehydrogenase, aspartate and alanine aminotransferase, prothrombin time, and fibrinogen levels compared to patients with cerebrovascular disease but without COVID-19 (table 2).
In univariable analysis, increased age, higher qSOFA scores, thrombocytopenia, and elevated C-reactive protein and lactate dehydrogenase were associated with in-hospital death in the COVID-19 group (table 3). In the multivariable logistic regression model, we found that higher qSOFA scores (odds ratio $4.47,95 \%$ CI $1.21-16.50, p=0.025)$, lower platelets (odds ratio 0.98, 95\% CI 0.97-0.99, $p=0.005$ ), and higher lactate dehydrogenase (odds ratio 1.01, 95\% CI 1.00-1.03, $p=$ 0.009 ) on admission were all associated with increased odds of death in patients with COVID-19 (table 3).

\section{Discussion}

This retrospective cohort study identified several differences between patients with and without COVID-19 who were hospitalized for a neurologic disease. In particular, patients with COVID-19 were older, had higher qSOFA scores on admission, and had a higher rate of cerebrovascular disorders compared to patients without COVID-19. During hospitalization, patients with COVID-19 had a higher incidence of delirium and fever, with prolonged hospital length of stay and increased in-hospital mortality rates. In addition, patients with COVID-19 had significant differences in laboratory values on admission, including blood count analysis, acute-phase proteins, and coagulation profiles.

We identified potential risk factors for a poor prognosis at an early stage such as high qSOFA score, thrombocytopenia, and increased lactate dehydrogenase levels. Previous reports have also found that SOFA and qSOFA scores were associated with in-hospital mortality, as well as d-dimer and older age, in adult inpatients with COVID-19. ${ }^{3}$ The qSOFA is a bedside prompt that may identify patients with suspected infection who are at greater risk for a poor outcome outside the intensive care unit. 
Table 1 Demographic, clinical, laboratory characteristics, treatment, and clinical outcomes of all included patients

\begin{tabular}{|c|c|c|c|c|}
\hline & Total $(n=173)$ & Non-COVID-19 ( $n=117)$ & COVID-19 $(n=56)$ & $p$ Value \\
\hline \multicolumn{5}{|l|}{ Demographic and clinical characteristics } \\
\hline Age, y & $72.3(57.5-80.5)$ & $70.1(52.9-78.6)$ & $77.0(67.0-83.8)$ & 0.006 \\
\hline Sex, $n(\%)$ & & & & 0.633 \\
\hline Female & $80(46.2)$ & $52(44.4)$ & $28(50.0)$ & \\
\hline Male & $93(53.8)$ & $65(55.6)$ & $28(50.0)$ & \\
\hline Current smoker, n (\%) & $8(4.6)$ & $7(6.0)$ & $1(1.8)$ & 0.605 \\
\hline \multicolumn{5}{|l|}{ Comorbidity, n (\%) } \\
\hline Diabetes mellitus & 34 (19.7) & $20(17.1)$ & $14(25.0)$ & 0.434 \\
\hline Hypercholesterolemia & $47(27.2)$ & 35 (29.9) & $12(21.4)$ & 0.434 \\
\hline Hypertension & $108(62.4)$ & $77(65.8)$ & $31(55.4)$ & 0.434 \\
\hline Coronary heart disease & $20(11.6)$ & $11(9.4)$ & $9(16.1)$ & 0.434 \\
\hline Malignancy & $43(24.9)$ & 35 (29.9) & $8(14.3)$ & 0.139 \\
\hline Chronic kidney disease & $7(4.0)$ & $5(4.3)$ & $2(3.6)$ & 1.000 \\
\hline Immunodeficiency & $5(2.9)$ & $4(3.4)$ & $1(1.8)$ & 1.000 \\
\hline qSOFA score & $0.7(0.6-0.7)$ & $0.5(0.4-0.6)$ & $0.9(0.7-1.1)$ & 0.006 \\
\hline Admitting neurologic diagnosis, $n$ (\%) & & & & 0.035 \\
\hline Cerebrovascular disease & $111(64.2)$ & $68(58.1)$ & $43(76.8)$ & \\
\hline Epilepsy & $23(13.3)$ & $19(16.2)$ & $4(7.1)$ & \\
\hline Inflammatory/infectious disease & $9(5.2)$ & $9(7.7)$ & $0(0.0)$ & \\
\hline Neoplastic & $3(1.7)$ & $3(2.6)$ & $0(0.0)$ & \\
\hline Other & $27(15.6)$ & $18(15.4)$ & $9(16.1)$ & \\
\hline \multicolumn{5}{|l|}{ Laboratory findings } \\
\hline White cell count, $\mathrm{mm}^{3}$ & $8.4(6.6-10.6)$ & $8.2(6.6-10.5)$ & $8.7(6.7-12.9)$ & 0.334 \\
\hline Neutrophil count, $\mathrm{mm}^{3}$ & $5.9(4.1-8.2)$ & $5.5(3.9-8.1)$ & $7.1(4.9-9.4)$ & 0.024 \\
\hline Lymphocyte count, $\mathrm{mm}^{3}$ & $1.4(1.0-1.9)$ & $1.6(1.2-2.1)$ & $1.0(0.8-1.4)$ & $<0.001$ \\
\hline Platelet count, $\mathrm{mm}^{3}$ & $228.0(178.5-290.5)$ & $215.0(175.0-266.0)$ & $262.6(191.8-311.8)$ & 0.021 \\
\hline Hemoglobin, g/dL & $13.3(11.9-14.5)$ & $13.2(11.8-14.5)$ & $13.4(12.2-14.7)$ & 0.337 \\
\hline C-reactive protein, $\mathrm{mg} / \mathrm{L}$ & $7.4(1.8-32.4)$ & $5.3(1.2-14.9)$ & $28.9(4.5-70.5)$ & $<0.001$ \\
\hline Erythrocyte sedimentation rate, $\mathrm{mm} / 1 \mathrm{~h}$ & $25.0(9.5-31.0)$ & $20.0(13.0-31.3)$ & $53.5(30.0-84.8)$ & $<0.001$ \\
\hline High-sensitivity troponin $\mathrm{T}, \mathrm{ng} / \mathrm{L}$ & $16.0(9.5-31.0)$ & $14.5(7.8-19.8)$ & $27.0(13.0-41.0)$ & 0.015 \\
\hline Lactate dehydrogenase, U/L & $204.9(168.0-296.5)$ & $184.0(161.0-214.0)$ & $275.7(232.5-369.5)$ & $<0.001$ \\
\hline Aspartate aminotransferase, $\mathrm{U} / \mathrm{L}$ & $26.0(21.0-38.0)$ & $24.0(20.0-32.0)$ & $37.5(24.5-60.8)$ & $<0.001$ \\
\hline Alanine aminotransferase, U/L & $24.0(17.0-36.0)$ & $22.0(15.5-31.0)$ & $32.5(20.0-43.2)$ & $<0.001$ \\
\hline Total bilirubin, $\mu \mathrm{mol} / \mathrm{L}$ & $0.60(0.47-0.85)$ & $0.60(0.47-0.83)$ & $0.60(0.46-0.98)$ & 0.592 \\
\hline Creatine kinase, U/L & $112.0(65.0-251.5)$ & $109.0(64.5-237.5)$ & $117.0(65.8-343.0)$ & 0.312 \\
\hline Creatinine, $\mu \mathrm{mol} / \mathrm{L}$ & $0.91(0.77-1.10)$ & $0.91(0.76-1.10)$ & $0.94(0.77-1.11)$ & 0.507 \\
\hline Prothrombin time, $\mathbf{s}$ & $11.8(12.3-13.3)$ & $12.2(11.6-12.9)$ & $12.7(12.1-15.2)$ & 0.005 \\
\hline
\end{tabular}


Table 1 Demographic, clinical, laboratory characteristics, treatment, and clinical outcomes of all included patients (continued)

\begin{tabular}{|c|c|c|c|c|}
\hline & Total $(n=173)$ & Non-COVID-19 $(n=117)$ & COVID-19 $(n=56)$ & $p$ Value \\
\hline Activated thromboplastin time, $s$ & $29.3(27.3-31.2)$ & $29.3(27.5-31.3)$ & $29.4(27.3-31.1)$ & 0.959 \\
\hline D-dimer, mg/L & $680.5(313.3-2,239.5)$ & $317.0(200.0-913.0)$ & $735.5(329.8-2,710.5)$ & 0.285 \\
\hline Fibrinogen, $\mathrm{mg} / \mathrm{dL}$ & $348.4(298.5-461.1)$ & $321.0(288.5-376.1)$ & $484.0(376.3-600.3)$ & $<0.001$ \\
\hline Serum ferritin, $\mu g / L$ & $377.0(173.5-706.5)$ & $155.0(66.5-340.25)$ & $392.0(194.5-827.0)$ & 0.119 \\
\hline \multicolumn{5}{|l|}{ Treatments and examinations } \\
\hline Antibiotic treatment, $\mathrm{n}(\%)$ & $55(31.8)$ & $19(16.2)$ & $36(64.3)$ & $<0.001$ \\
\hline Antiviral treatment, n (\%) & $40(23.1)$ & $2(1.7)$ & $38(67.9)$ & $<0.001$ \\
\hline High-flow oxygen therapy, $n$ (\%) & $54(31.2)$ & $11(9.4)$ & $43(76.8)$ & $<0.001$ \\
\hline Diagnostic tests, $n$ & $5.0(4.0-7.0)$ & $5.0(3.0-7.0)$ & $5.0(4.0-8.0)$ & 0.553 \\
\hline \multicolumn{5}{|l|}{ Outcomes } \\
\hline In hospital mortality, n (\%) & $26(15.0)$ & $5(4.3)$ & $21(37.5)$ & $<0.001$ \\
\hline Incident delirium, n (\%) & 24 (13.9) & $9(7.7)$ & $15(26.8)$ & 0.003 \\
\hline Fever during hospitalization, $\mathrm{n}(\%)$ & $41(23.7)$ & $14(12.0)$ & $27(48.2)$ & $<0.001$ \\
\hline Hospital length of stay, d & $5.0(4.0-8.0)$ & $5.0(4.0-8.0)$ & $6.0(3.3-10.0)$ & 0.424 \\
\hline mRS score premorbid & $1.0(0.0-2.0)$ & $1.0(0.0-2.0)$ & $1.0(1.0-2.0)$ & 0.903 \\
\hline mRS score discharge & $2.0(1.0-4.0)$ & $2.0(1.0-3.0)$ & $5.0(2.3-6.0)$ & $<0.001$ \\
\hline
\end{tabular}

Abbreviations: COVID-19 = coronavirus disease 2019; mRS = modified Rankin Scale; qSOFA = quick Sequential Organ Failure Assessment.

Data are median (interquartile range), or number (percent). The $p$ values were calculated by the Mann-Whitney $U$ test, $\chi^{2}$ test or Fisher exact test as appropriate and corrected for multiple comparisons with the Benjamini-Hochberg false discovery rate.

qSOFA can be performed rapidly by the clinician without the need for laboratory analysis. ${ }^{12}$

We observed a significant increase of stroke rates in patients with COVID-19, with worse outcomes compared to the group without COVID-19, including higher mRS scores at discharge and a significantly lower number of patients with a good outcome, at par with access to acutephase therapies. As recently suggested in a statement by the American Heart Association/American Stroke Association Stroke Council Leadership, stroke mechanisms in COVID19 could include different processes, including the release of proinflammatory cytokines with a direct effect on plaque rupture through local inflammation and activation of coagulation factors or cardioembolism from virus-related cardiac injury. ${ }^{13-17}$

Moreover, a direct effect of the virus on endothelial cells or on heart tissue could be hypothesized, considering that the receptor for SARS-CoV-2, the angiotensin-converting enzyme $2,{ }^{18-20}$ is expressed on vascular endothelial cells and myocytes. ${ }^{21-23}$

In our cohort, we observed several indexes of an altered coagulability in patients with COVID-19 compared to patients without COVID-19. Prothrombin time and d-dimer were increased in the former group, as well as inflammatory indexes such as C-reactive protein and erythrocyte sedimentation rate. This marker profile is consistent with what has been observed in disseminated intravascular coagulation and may play an important role in stroke incidence and severity in patients with COVID-19. ${ }^{24}$ Abnormal coagulation parameters have also been shown to be associated with poor prognosis in patients with COVID-19-associated pneumonia. 25

From the present analysis, patients who underwent endovascular therapy or IV fibrinolysis in the COVID-19 group had more severe outcomes, including increases in NIHSS and mRS scores at discharge. This raises a critical issue of whether patients with COVID-19 should be equally treated with acute-phase therapies; additional studies on broader populations should try to shed further light on this matter.

What also emerges from this study is that differences in laboratory and most clinical features between neurology admissions of patients with and without COVID-19 mostly reflect the features of COVID-19 infection, including fever, thrombocytopenia, elevated lactate dehydrogenase, and high morbidity and mortality. ${ }^{3,9,26,27}$ The concomitant neurologic comorbidity such as stroke or other major neurologic 
Table 2 Demographic, clinical, laboratory characteristics, treatment, and clinical outcomes of patients with cerebrovascular disease

\begin{tabular}{|c|c|c|c|c|}
\hline & Total $(n=111)$ & Non-COVID-19 $(n=68)$ & COVID-19 $(n=43)$ & $p$ Value \\
\hline Cerebrovascular event, $\mathbf{n}(\%)$ & & & & 0.560 \\
\hline TIA & $13(11.7)$ & $8(11.8)$ & $5(11.6)$ & \\
\hline Ischemic stroke & $85(76.6)$ & $50(73.5)$ & $35(81.4)$ & \\
\hline Hemorrhagic stroke & $13(11.7)$ & $10(14.7)$ & $3(7.0)$ & \\
\hline \multicolumn{5}{|l|}{ Demographic and clinical characteristics } \\
\hline Age, y & $76.3(61.4-82.5)$ & $74.8(58.3-80.4)$ & $76.9(66.8-85.2)$ & 0.204 \\
\hline Sex, n (\%) & & & & 0.765 \\
\hline Female & $49(44.1)$ & $28(41.2)$ & $21(48.8)$ & \\
\hline Male & $62(55.9)$ & $40(58.8)$ & $22(51.2)$ & \\
\hline Current smoker, n (\%) & $7(6.3)$ & $6(8.8)$ & $1(2.3)$ & 0.526 \\
\hline \multicolumn{5}{|l|}{ Comorbidity, n (\%) } \\
\hline Diabetes mellitus & $24(21.6)$ & $14(20.6)$ & $10(23.3)$ & 0.781 \\
\hline Hypercholesterolemia & $33(29.7)$ & $23(33.8)$ & $10(23.3)$ & 0.526 \\
\hline Hypertension & $77(69.4)$ & $51(75.0)$ & $26(60.5)$ & 0.388 \\
\hline Coronary heart disease & $16(14.4)$ & $8(11.8)$ & $8(18.6)$ & 0.643 \\
\hline Malignancy & $24(21.6)$ & 19 (27.9) & $5(11.6)$ & 0.220 \\
\hline Chronic kidney disease & $5(4.5)$ & $3(4.4)$ & $2(4.7)$ & 1.000 \\
\hline Immunodeficiency & $4(3.6)$ & $3(4.4)$ & $1(2.3)$ & 1.000 \\
\hline qSOFA score & $0.7(0.6-0.8)$ & $0.5(0.4-0.7)$ & $0.9(0.7-1.1)$ & 0.077 \\
\hline \multicolumn{5}{|l|}{ Laboratory findings } \\
\hline White cell count, $\mathrm{mm}^{3}$ & $8.5(6.6-10.6)$ & $8.3(6.6-10.5)$ & $8.9(6.7-11.7)$ & 0.424 \\
\hline Neutrophil count, $\mathrm{mm}^{3}$ & $6.1(4.5-8.5)$ & $5.5(4.1-8.1)$ & $7.3(5.0-9.6)$ & 0.067 \\
\hline Lymphocyte count, $\mathrm{mm}^{3}$ & $1.3(0.9-1.8)$ & $1.5(1.2-2.1)$ & $1.0(0.8-1.4)$ & $<0.001$ \\
\hline Platelet count, $\mathrm{mm}^{3}$ & $231.0(181.0-294.0)$ & 211.5 (174.5-264.3) & $276.0(197.0-315.0)$ & 0.036 \\
\hline Hemoglobin, g/dL & $13.2(11.5-14.6)$ & $12.7(11.5-14.4)$ & $13.4(12.1-14.9)$ & 0.133 \\
\hline C-reactive protein, $\mathrm{mg} / \mathrm{L}$ & $8.1(2.0-35.7)$ & $5.1(1.5-12.9)$ & $28.0(4.4-71.1)$ & $<0.001$ \\
\hline Erythrocyte sedimentation rate, $\mathrm{mm} / 1 \mathrm{~h}$ & $25.0(14.0-50.0)$ & $19.5(12.3-30.8)$ & $49.0(29.3-85.0)$ & $<0.001$ \\
\hline High-sensitivity troponin $\mathrm{T}$, ng/L & $16.0(10.0-29.0)$ & $16.0(9.3-21.3)$ & $27.0(10.0-41.0)$ & 0.067 \\
\hline Lactate dehydrogenase, U/L & $220.0(168.0-277.5)$ & $177.0(156.8-221.5)$ & $277.7(239.0-365.0)$ & $<0.001$ \\
\hline Aspartate aminotransferase, $\mathrm{U} / \mathrm{L}$ & $27.7(21.0-38.0)$ & $24.0(21.0-30.5)$ & $36.0(28.0-52.0)$ & $<0.001$ \\
\hline Alanine aminotransferase, U/L & $25.0(16.0-35.0)$ & $20.5(15.0-27.8)$ & $31.0(20.0-40.7)$ & $<0.001$ \\
\hline Total bilirubin, $\mu \mathrm{mol} / \mathrm{L}$ & $0.64(0.48-0.94)$ & $0.64(0.48-0.91)$ & $0.66(0.48-1.0)$ & 0.424 \\
\hline Creatine kinase, U/L & $109.0(66.0-241.0)$ & $104.5(65.3-221.5)$ & $114.0(69.0-337.0)$ & 0.424 \\
\hline Creatinine, $\mu \mathrm{mol} / \mathrm{L}$ & $0.95(0.77-1.14)$ & $0.95(0.77-1.14)$ & $0.95(0.77-1.11)$ & 0.864 \\
\hline Prothrombin time, $\mathbf{s}$ & $12.5(12.0-14.1)$ & $12.4(11.8-13.0)$ & $12.8(12.2-15.6)$ & 0.029 \\
\hline Activated thromboplastin time, $s$ & $29.0(26.8-31.0)$ & $29.1(26.7-31.4)$ & $28.6(27.3-30.7)$ & 0.770 \\
\hline D-dimer, mg/L & $693.0(325.0-2,868.0)$ & 317.0 (197.0-437.0) & $735.5(364.3-2,910.8)$ & 0.626 \\
\hline
\end{tabular}


Table 2 Demographic, clinical, laboratory characteristics, treatment, and clinical outcomes of patients with cerebrovascular disease (continued)

\begin{tabular}{|c|c|c|c|c|}
\hline & Total $(n=111)$ & Non-COVID-19 $(n=68)$ & COVID-19 $(n=43)$ & $p$ Value \\
\hline Fibrinogen, mg/dL & $351.0(291.0-466.0)$ & $312.0(261.1-386.4)$ & $471.0(368.0-545.0)$ & $<0.001$ \\
\hline Serum ferritin, $\mu \mathrm{g} / \mathrm{L}$ & $411.0(244.0-739.0)$ & $152.0(38.0-266.0)$ & $452.5(268.0-871.0)$ & 0.130 \\
\hline \multicolumn{5}{|l|}{ Treatments and examinations } \\
\hline Antibiotic treatment, $\mathrm{n}(\%)$ & $39(35.1)$ & $11(16.2)$ & $28(65.1)$ & $<0.001$ \\
\hline Antiviral treatment, n (\%) & $27(24.3)$ & $0(0.0)$ & $27(62.8)$ & $<0.001$ \\
\hline High-flow oxygen therapy, n (\%) & $40(36.0)$ & $7(10.3)$ & $33(76.7)$ & $<0.001$ \\
\hline Diagnostic tests, $n$ & $6.0(4.0-8.0)$ & $6.0(5.0-8.0)$ & $6.0(4.0-8.0)$ & \\
\hline Acute-phase therapies (ischemic stroke only), $n$ (\%) & $22(25.9)$ & $13(26.0)$ & $9(25.7)$ & 1.000 \\
\hline IV fibrinolysis & $6(6.1)$ & $2(3.4)$ & $4(10.0)$ & 0.384 \\
\hline Endovascular therapy & $10(10.2)$ & $8(13.8)$ & $2(5.0)$ & 0.785 \\
\hline Bridging therapy & $6(7.1)$ & $3(6.0)$ & $3(8.6)$ & 0.785 \\
\hline \multicolumn{5}{|l|}{ Outcomes } \\
\hline In hospital mortality, n (\%) & $19(17.1)$ & $4(5.9)$ & $15(34.9)$ & $<0.001$ \\
\hline Incident delirium, n (\%) & $13(11.7)$ & $4(5.9)$ & $9(20.9)$ & 0.047 \\
\hline Fever during hospitalization, $\mathbf{n}(\%)$ & $29(26.1)$ & $8(11.8)$ & $21(48.8)$ & $<0.001$ \\
\hline Hospital length of stay, d & $5.0(4.0-8.0)$ & $5.0(4.0-8.0)$ & $6.0(4.0-9.0)$ & 0.425 \\
\hline mRS score premorbid & $1.0(0.0-2.0)$ & $1.0(0.0-2.0)$ & $1.0(0.0-1.0)$ & 0.727 \\
\hline mRS score discharge & $2.0(1.0-5.0)$ & $2.0(1.0-3.0)$ & $5.0(2.0-6.0)$ & $<0.001$ \\
\hline Good outcome & $59(53.2)$ & $48(70.6)$ & $11(25.6)$ & $<0.001$ \\
\hline NIHSS admission & $5.0(2.0-15.0)$ & $4.0(2.0-14.8)$ & $10.0(3.0-15.0)$ & 0.147 \\
\hline NIHSS discharge & $3.0(0.0-10.0)$ & $2.0(0.0-6.8)$ & $9.0(1.0-19.0)$ & 0.005 \\
\hline
\end{tabular}

Abbreviations: $\mathrm{mRS}$ = modified Rankin Scale; NIHSS = NIH Stroke Scale; qSOFA = quick Sequential Organ Failure Assessment.

Bridging therapy is IV fibrinolysis followed by endovascular therapy. Data are median (interquartile range) or number (percent). The $p$ values were calculated by the Mann-Whitney $U$ test, $\chi^{2}$ test, or Fisher exact test as appropriate and corrected for multiple comparisons with the Benjamini-Hochberg false discovery rate.

disorder could possibly increase even further the high mortality observed in this patient group. Moreover, it is still not clear whether COVID-19 may increase the incidence of stroke $^{28}$ and other neurologic diseases such as encephalitis ${ }^{29}$ or immune-mediated neurologic disorders. ${ }^{30-32}$

With the surge of intensive care unit admissions during the pandemic $^{2}$ and as underlined by the American Heart Association/American Stroke Association Stroke Council Leadership, it has become necessary to prioritize intensive care unit resources, at the possible expenses of patients with stroke, ${ }^{15,33}$ withholding ventilation when necessary and raising issues on potential legal liabilities. ${ }^{34}$ These aspects should be addressed in neurology units that deal with acutephase diseases and patients with COVID-19.

We acknowledge that this study has some limitations. First, due to the retrospective study design, not all laboratory tests were performed in all patients, including highsensitivity troponin $\mathrm{T}$, ferritin, and $\mathrm{d}$-dimer. Therefore, their role could have not been thoroughly assessed in this study. Second, results on stroke do not take into account several factors due to the retrospective design, including stroke subtypes, infarct volume, and recanalization rates. Third, interpretation of our findings could be limited by sample size and by the single-center design. Fourth, there could be a selection bias due to the unwillingness of patients with COVID-related symptoms or infection to come for hospital neurologic evaluation unless extremely necessary such as stroke, epilepsy, or other major neurologic disorders. Additional larger, multicenter, prospective studies should be performed to confirm these findings.

To the best of our knowledge, this is the largest retrospective cohort study among patients with neurologic 
Table 3 Risk factors on admission associated with in-hospital mortality in patients with COVID-19

\begin{tabular}{|c|c|c|c|c|}
\hline & Univariable OR $(95 \% \mathrm{Cl})$ & $p$ Value & Multivariable OR $(95 \% \mathrm{Cl})$ & $p$ Value \\
\hline \multicolumn{5}{|c|}{ Demographic and clinical characteristics } \\
\hline Age, y & $1.06(1.00-1.12)$ & 0.043 & $1.08(0.99-1.18)$ & 0.090 \\
\hline Female sex (vs male) & $0.11(0.11-1.03)$ & 0.057 & & \\
\hline Current smoker (vs nonsmoker) & 0.000 & 1.000 & & \\
\hline \multicolumn{5}{|c|}{ Comorbidity present (vs not present) } \\
\hline Diabetes mellitus & $0.36(0.09-1.50)$ & 0.161 & & \\
\hline Hypercholesterolemia & $0.48(0.11-2.03)$ & 0.319 & & \\
\hline Hypertension & $0.32(0.10-0.99)$ & 0.048 & & \\
\hline Coronary heart disease & $0.81(0.18-3.63)$ & 0.778 & & \\
\hline Malignancy & $1.00(0.21-4.69)$ & 1.000 & & \\
\hline Chronic kidney disease & $1.70(0.10-28.70)$ & 0.713 & & \\
\hline Immunodeficiency & 0.000 & 1.000 & & \\
\hline qSOFA score & $5.12(1.77-14.83)$ & 0.003 & $4.47(1.21-16.50)$ & 0.025 \\
\hline \multicolumn{5}{|l|}{ Laboratory findings } \\
\hline White cell count & $1.02(0.93-1.11)$ & 0.730 & & \\
\hline Neutrophil count & $1.02(0.93-1.13)$ & 0.651 & & \\
\hline Lymphocyte count & $0.45(0.13-1.50)$ & 0.192 & & \\
\hline Platelet count per $1 \mathrm{~mm}^{3}$ & $0.99(0.98-0.99)$ & 0.012 & $0.98(0.97-0.99)$ & 0.005 \\
\hline Hemoglobin & $1.18(0.86-1.61)$ & 0.303 & & \\
\hline C-reactive protein & $1.01(1.00-1.02)$ & 0.031 & $1.00(0.99-1.01)$ & 0.981 \\
\hline Erythrocyte sedimentation rate & $1.01(0.99-1.03)$ & 0.460 & & \\
\hline High-sensitivity troponin $\mathrm{T}$ & $1.03(1.00-1.07)$ & 0.078 & & \\
\hline Lactate dehydrogenase & $1.01(1.00-1.02)$ & 0.004 & $1.01(1.00-1.03)$ & 0.009 \\
\hline Aspartate aminotransferase & $1.02(1.00-1.03)$ & 0.112 & & \\
\hline Alanine aminotransferase & $1.00(0.99-1.02)$ & 0.599 & & \\
\hline Total bilirubin & $0.79(0.31-2.03)$ & 0.630 & & \\
\hline Creatine kinase & $1.00(1.00-1.00)$ & 0.080 & & \\
\hline Creatinine & $4.71(0.88-25.3)$ & 0.071 & & \\
\hline Prothrombin time & $1.07(0.96-1.20)$ & 0.210 & & \\
\hline Activated thromboplastin time & $1.02(0.91-1.15)$ & 0.682 & & \\
\hline D-dimer & $1.00(1.00-1.00)$ & 0.058 & & \\
\hline Fibrinogen & $1.00(1.00-1.01)$ & 0.108 & & \\
\hline Serum ferritin & $1.00(1.00-1.00)$ & 0.773 & & \\
\hline
\end{tabular}

Abbreviations: $\mathrm{Cl}$ = confidence interval; COVID-19 = coronavirus disease 2019; OR = odds ratio; qSOFA = quick Sequential Organ Failure Assessment.

disorders and COVID-19 with a definite outcome. Patients with COVID-19 and neurologic disease have worse clinical and neurologic outcomes with higher in-hospital mortality rates compared to patients without COVID-19.

\section{Acknowledgment}

The authors express their gratitude to the nurses, auxiliary staff, technicians, and all the colleagues who collaborated for the management of patients during the COVID-19 outbreak. 
Moreover, they thank Dr. Marco Trivelli, Dr. Camilo Rossi, and Dr. Loretta Jacquot for their support in setting up a neuro-COVID unit.

\section{Study funding}

No targeted funding reported.

\section{Disclosure}

A. Benussi, A. Pilotto, E. Premi, I. Libri, M. Giunta, C. Agosti, A. Alberici, E. Baldelli, M. Benini, S. Bonacina, L. Brambilla, S. Caratozzolo, M. Cortinovis, A. Costa, S. Cotti Piccinelli, E. Cottini, V. Cristillo, I. Delrio, M. Filosto, M. Gamba, S. Gazzina, N. Gilberti, S. Gipponi, A. Imarisio, P. Invernizzi, U. Leggio, M. Leonardi, P. Liberini, M. Locatelli, S. Masciocchi, L. Poli, R. Rao, B. Risi, L. Rozzini, A. Scalvini, F. Schiano di Cola, R. Spezi, V. Vergani, I. Volonghi, N. Zoppi, B. Borroni, M. Magoni, and A. Pezzini report no disclosures relevant to the manuscript. A. Padovani is consultant and served on the scientific advisory board of GE Healthcare, Eli-Lilly, and Actelion Ltd Pharmaceuticals and received speaker honoraria from Nutricia, PIAM, Lansgstone Technology, GE Healthcare, Lilly, UCB Pharma, and Chiesi Pharmaceuticals. Go to Neurology.org/N for full disclosures.

\section{Publication history}

Received by Neurology April 18, 2020. Accepted in final form May 13, 2020.

Appendix Authors

\begin{tabular}{lll}
\hline Name & Location & Contribution \\
\hline $\begin{array}{ll}\text { Alberto } \\
\text { Benussi, MD }\end{array}$ & $\begin{array}{l}\text { University of Brescia, } \\
\text { Italy }\end{array}$ & $\begin{array}{l}\text { Designed and } \\
\text { conceptualized study; major } \\
\text { role in the acquisition of } \\
\text { data; analyzed the data; } \\
\text { drafted the manuscript for } \\
\text { intellectual content }\end{array}$ \\
\end{tabular}

\begin{tabular}{lll}
\hline $\begin{array}{l}\text { Andrea } \\
\text { Pilotto, MD }\end{array}$ & $\begin{array}{l}\text { University of Brescia, } \\
\text { Italy }\end{array}$ & $\begin{array}{l}\text { Major role in the acquisition } \\
\text { of data; analyzed the data; } \\
\text { revised the manuscript for } \\
\text { intellectual content }\end{array}$ \\
\hline $\begin{array}{l}\text { Enrico Premi, } \\
\text { MD }\end{array}$ & $\begin{array}{l}\text { ASST Spedali Civili, } \\
\text { Brescia, Italy }\end{array}$ & $\begin{array}{l}\text { Interpreted the data; } \\
\text { analyzed the data; revised } \\
\text { the manuscript for } \\
\text { intellectual content }\end{array}$ \\
\hline $\begin{array}{l}\text { Ilenia Libri, } \\
\text { MD }\end{array}$ & $\begin{array}{l}\text { University of Brescia, } \\
\text { Italy }\end{array}$ & $\begin{array}{l}\text { Major role in the acquisition } \\
\text { of data; revised the } \\
\text { manuscript for intellectual } \\
\text { content }\end{array}$ \\
\hline $\begin{array}{l}\text { Marcello } \\
\text { Giunta, MD }\end{array}$ & $\begin{array}{ll}\text { University of Brescia, } \\
\text { Italy }\end{array}$ & $\begin{array}{l}\text { Major role in the acquisition } \\
\text { of data; revised the } \\
\text { manuscript for intellectual } \\
\text { content }\end{array}$ \\
\hline $\begin{array}{l}\text { Alberici, MD } \\
\text { MDiara Agosti, }\end{array}$ & $\begin{array}{l}\text { ASST Spedali Civili, } \\
\text { Brescia, Italy }\end{array}$ & $\begin{array}{l}\text { Interpreted the data; revised } \\
\text { the manuscript for } \\
\text { intellectual content }\end{array}$ \\
\hline
\end{tabular}

Appendix (continued)

\begin{tabular}{|c|c|c|}
\hline Name & Location & Contribution \\
\hline $\begin{array}{l}\text { Enrico } \\
\text { Baldelli, MD }\end{array}$ & $\begin{array}{l}\text { University of Brescia, } \\
\text { Italy }\end{array}$ & $\begin{array}{l}\text { Interpreted the data; revised } \\
\text { the manuscript for } \\
\text { intellectual content }\end{array}$ \\
\hline $\begin{array}{l}\text { Matteo } \\
\text { Benini, MD }\end{array}$ & $\begin{array}{l}\text { University of Bologna, } \\
\text { Italy }\end{array}$ & $\begin{array}{l}\text { Interpreted the data; revised } \\
\text { the manuscript for } \\
\text { intellectual content }\end{array}$ \\
\hline $\begin{array}{l}\text { Sonia } \\
\text { Bonacina, MD }\end{array}$ & $\begin{array}{l}\text { University of Brescia, } \\
\text { Italy }\end{array}$ & $\begin{array}{l}\text { Interpreted the data; revised } \\
\text { the manuscript for } \\
\text { intellectual content }\end{array}$ \\
\hline $\begin{array}{l}\text { Laura } \\
\text { Brambilla, } \\
\text { MD }\end{array}$ & $\begin{array}{l}\text { Foundation IRCCS } \\
\text { Neurologic Institute } \\
\text { Carlo Besta, Milan, Italy }\end{array}$ & $\begin{array}{l}\text { Interpreted the data; revised } \\
\text { the manuscript for } \\
\text { intellectual content }\end{array}$ \\
\hline $\begin{array}{l}\text { Salvatore } \\
\text { Caratozzolo, } \\
\text { MD }\end{array}$ & $\begin{array}{l}\text { ASST Spedali Civili, } \\
\text { Brescia, Italy }\end{array}$ & $\begin{array}{l}\text { Interpreted the data; revised } \\
\text { the manuscript for } \\
\text { intellectual content }\end{array}$ \\
\hline $\begin{array}{l}\text { Matteo } \\
\text { Cortinovis, } \\
\text { MD }\end{array}$ & $\begin{array}{l}\text { University of Brescia, } \\
\text { Italy }\end{array}$ & $\begin{array}{l}\text { Interpreted the data; revised } \\
\text { the manuscript for } \\
\text { intellectual content }\end{array}$ \\
\hline $\begin{array}{l}\text { Angelo Costa, } \\
\text { MD }\end{array}$ & $\begin{array}{l}\text { ASST Spedali Civili, } \\
\text { Brescia, Italy }\end{array}$ & $\begin{array}{l}\text { Interpreted the data; revised } \\
\text { the manuscript for } \\
\text { intellectual content }\end{array}$ \\
\hline $\begin{array}{l}\text { Stefano Cotti } \\
\text { Piccinelli, MD }\end{array}$ & $\begin{array}{l}\text { University of Brescia, } \\
\text { Italy }\end{array}$ & $\begin{array}{l}\text { Interpreted the data; revised } \\
\text { the manuscript for } \\
\text { intellectual content }\end{array}$ \\
\hline $\begin{array}{l}\text { Elisabetta } \\
\text { Cottini, MD }\end{array}$ & $\begin{array}{l}\text { ASST Spedali Civili, } \\
\text { Brescia, Italy }\end{array}$ & $\begin{array}{l}\text { Interpreted the data; revised } \\
\text { the manuscript for } \\
\text { intellectual content }\end{array}$ \\
\hline $\begin{array}{l}\text { Viviana } \\
\text { Cristillo, MD }\end{array}$ & $\begin{array}{l}\text { University of Brescia, } \\
\text { Italy }\end{array}$ & $\begin{array}{l}\text { Interpreted the data; revised } \\
\text { the manuscript for } \\
\text { intellectual content }\end{array}$ \\
\hline $\begin{array}{l}\text { Ilenia Delrio, } \\
\text { MD }\end{array}$ & $\begin{array}{l}\text { ASST Spedali Civili, } \\
\text { Brescia, Italy }\end{array}$ & $\begin{array}{l}\text { Interpreted the data; revised } \\
\text { the manuscript for } \\
\text { intellectual content }\end{array}$ \\
\hline $\begin{array}{l}\text { Massimiliano } \\
\text { Filosto, MD }\end{array}$ & $\begin{array}{l}\text { ASST Spedali Civili, } \\
\text { Brescia, Italy }\end{array}$ & $\begin{array}{l}\text { Interpreted the data; revised } \\
\text { the manuscript for } \\
\text { intellectual content }\end{array}$ \\
\hline $\begin{array}{l}\text { Massimo } \\
\text { Gamba, MD }\end{array}$ & $\begin{array}{l}\text { ASST Spedali Civili, } \\
\text { Brescia, Italy }\end{array}$ & $\begin{array}{l}\text { Interpreted the data; revised } \\
\text { the manuscript for } \\
\text { intellectual content }\end{array}$ \\
\hline $\begin{array}{l}\text { Stefano } \\
\text { Gazzina, MD }\end{array}$ & $\begin{array}{l}\text { ASST Spedali Civili, } \\
\text { Brescia, Italy }\end{array}$ & $\begin{array}{l}\text { Interpreted the data; revised } \\
\text { the manuscript for } \\
\text { intellectual content }\end{array}$ \\
\hline $\begin{array}{l}\text { Nicola } \\
\text { Gilberti, MD }\end{array}$ & $\begin{array}{l}\text { ASST Spedali Civili, } \\
\text { Brescia, Italy }\end{array}$ & $\begin{array}{l}\text { Interpreted the data; revised } \\
\text { the manuscript for } \\
\text { intellectual content }\end{array}$ \\
\hline $\begin{array}{l}\text { Stefano } \\
\text { Gipponi, MD }\end{array}$ & $\begin{array}{l}\text { ASST Spedali Civili, } \\
\text { Brescia, Italy }\end{array}$ & $\begin{array}{l}\text { Interpreted the data; revised } \\
\text { the manuscript for } \\
\text { intellectual content }\end{array}$ \\
\hline $\begin{array}{l}\text { Alberto } \\
\text { Imarisio, MD }\end{array}$ & $\begin{array}{l}\text { University of Brescia, } \\
\text { Italy }\end{array}$ & $\begin{array}{l}\text { Interpreted the data; revised } \\
\text { the manuscript for } \\
\text { intellectual content }\end{array}$ \\
\hline $\begin{array}{l}\text { Paolo } \\
\text { Invernizzi, } \\
\text { MD }\end{array}$ & $\begin{array}{l}\text { Fondazione } \\
\text { Poliambulanza } \\
\text { Hospital, Brescia, Italy }\end{array}$ & $\begin{array}{l}\text { Interpreted the data; revised } \\
\text { the manuscript for } \\
\text { intellectual content }\end{array}$ \\
\hline $\begin{array}{l}\text { Ugo Leggio, } \\
\text { MD }\end{array}$ & $\begin{array}{l}\text { ASST Spedali Civili, } \\
\text { Brescia, Italy }\end{array}$ & $\begin{array}{l}\text { Interpreted the data; revised } \\
\text { the manuscript for } \\
\text { intellectual content }\end{array}$ \\
\hline
\end{tabular}


Appendix (continued)

\begin{tabular}{|c|c|c|}
\hline Name & Location & Contribution \\
\hline $\begin{array}{l}\text { Matilde } \\
\text { Leonardi, MD }\end{array}$ & $\begin{array}{l}\text { Foundation IRCCS } \\
\text { Neurologic Institute } \\
\text { Carlo Besta, Milan, Italy }\end{array}$ & $\begin{array}{l}\text { Interpreted the data; revised } \\
\text { the manuscript for } \\
\text { intellectual content }\end{array}$ \\
\hline $\begin{array}{l}\text { Paolo } \\
\text { Liberini, MD }\end{array}$ & $\begin{array}{l}\text { ASST Spedali Civili, } \\
\text { Brescia, Italy }\end{array}$ & $\begin{array}{l}\text { Interpreted the data; revised } \\
\text { the manuscript for } \\
\text { intellectual content }\end{array}$ \\
\hline $\begin{array}{l}\text { Martina } \\
\text { Locatelli, MD }\end{array}$ & $\begin{array}{l}\text { University of Brescia, } \\
\text { Italy }\end{array}$ & $\begin{array}{l}\text { Interpreted the data; revised } \\
\text { the manuscript for } \\
\text { intellectual content }\end{array}$ \\
\hline $\begin{array}{l}\text { Stefano } \\
\text { Masciocchi, } \\
\text { MD }\end{array}$ & $\begin{array}{l}\text { University of Brescia, } \\
\text { Italy }\end{array}$ & $\begin{array}{l}\text { Interpreted the data; revised } \\
\text { the manuscript for } \\
\text { intellectual content }\end{array}$ \\
\hline Loris Poli, MD & $\begin{array}{l}\text { ASST Spedali Civili, } \\
\text { Brescia, Italy }\end{array}$ & $\begin{array}{l}\text { Interpreted the data; revised } \\
\text { the manuscript for } \\
\text { intellectual content }\end{array}$ \\
\hline $\begin{array}{l}\text { Renata Rao, } \\
\text { MD }\end{array}$ & $\begin{array}{l}\text { ASST Spedali Civili, } \\
\text { Brescia, Italy }\end{array}$ & $\begin{array}{l}\text { Interpreted the data; } \\
\text { revised the manuscript } \\
\text { for intellectual content }\end{array}$ \\
\hline $\begin{array}{l}\text { Barbara Risi, } \\
\text { MD }\end{array}$ & $\begin{array}{l}\text { University of Brescia, } \\
\text { Italy }\end{array}$ & $\begin{array}{l}\text { Interpreted the data; revised } \\
\text { the manuscript for } \\
\text { intellectual content }\end{array}$ \\
\hline $\begin{array}{l}\text { Luca Rozzini, } \\
\text { MD }\end{array}$ & $\begin{array}{l}\text { University of Brescia, } \\
\text { Italy }\end{array}$ & $\begin{array}{l}\text { Interpreted the data; revised } \\
\text { the manuscript for } \\
\text { intellectual content }\end{array}$ \\
\hline $\begin{array}{l}\text { Andrea } \\
\text { Scalvini, MD }\end{array}$ & $\begin{array}{l}\text { University of Brescia, } \\
\text { Italy }\end{array}$ & $\begin{array}{l}\text { Interpreted the data; revised } \\
\text { the manuscript for } \\
\text { intellectual content }\end{array}$ \\
\hline $\begin{array}{l}\text { Francesca } \\
\text { Schiano di } \\
\text { Cola, MD }\end{array}$ & $\begin{array}{l}\text { University of Brescia, } \\
\text { Italy }\end{array}$ & $\begin{array}{l}\text { Interpreted the data; revised } \\
\text { the manuscript for } \\
\text { intellectual content }\end{array}$ \\
\hline $\begin{array}{l}\text { Raffaella } \\
\text { Spezi, MD }\end{array}$ & $\begin{array}{l}\text { ASST Spedali Civili, } \\
\text { Brescia, Italy }\end{array}$ & $\begin{array}{l}\text { Interpreted the data; revised } \\
\text { the manuscript for } \\
\text { intellectual content }\end{array}$ \\
\hline $\begin{array}{l}\text { Veronica } \\
\text { Vergani, MD }\end{array}$ & $\begin{array}{l}\text { ASST Spedali Civili, } \\
\text { Brescia, Italy }\end{array}$ & $\begin{array}{l}\text { Interpreted the data; revised } \\
\text { the manuscript for } \\
\text { intellectual content }\end{array}$ \\
\hline $\begin{array}{l}\text { Irene } \\
\text { Volonghi, MD }\end{array}$ & $\begin{array}{l}\text { ASST Spedali Civili, } \\
\text { Brescia, Italy }\end{array}$ & $\begin{array}{l}\text { Interpreted the data; revised } \\
\text { the manuscript for } \\
\text { intellectual content }\end{array}$ \\
\hline $\begin{array}{l}\text { Nicola Zoppi, } \\
\text { MD }\end{array}$ & $\begin{array}{l}\text { University of Brescia, } \\
\text { Italy }\end{array}$ & $\begin{array}{l}\text { Interpreted the data; revised } \\
\text { the manuscript for } \\
\text { intellectual content }\end{array}$ \\
\hline $\begin{array}{l}\text { Barbara } \\
\text { Borroni, MD }\end{array}$ & $\begin{array}{l}\text { University of Brescia, } \\
\text { Italy }\end{array}$ & $\begin{array}{l}\text { Designed and } \\
\text { conceptualized study; } \\
\text { revised the manuscript for } \\
\text { intellectual content }\end{array}$ \\
\hline $\begin{array}{l}\text { Mauro } \\
\text { Magoni, MD }\end{array}$ & $\begin{array}{l}\text { ASST Spedali Civili, } \\
\text { Brescia, Italy }\end{array}$ & $\begin{array}{l}\text { Interpreted the data; revised } \\
\text { the manuscript for } \\
\text { intellectual content }\end{array}$ \\
\hline $\begin{array}{l}\text { Alessandro } \\
\text { Pezzini, MD }\end{array}$ & $\begin{array}{l}\text { University of Brescia, } \\
\text { Italy }\end{array}$ & $\begin{array}{l}\text { Interpreted the data; revised } \\
\text { the manuscript for } \\
\text { intellectual content }\end{array}$ \\
\hline $\begin{array}{l}\text { Alessandro } \\
\text { Padovani, } \\
\text { MD, PhD }\end{array}$ & $\begin{array}{l}\text { University of Brescia, } \\
\text { Italy }\end{array}$ & $\begin{array}{l}\text { Designed and } \\
\text { conceptualized study; } \\
\text { drafted the manuscript for } \\
\text { intellectual content; } \\
\text { revised the manuscript for } \\
\text { intellectual content }\end{array}$ \\
\hline
\end{tabular}

\section{References}

1. Protezione Civile. Coronavirus. Available at: protezionecivile.gov.it/attivita-rischi/ rischio-sanitario/emergenze/coronavirus. Accessed April 5, 2020.

2. Grasselli G, Pesenti A, Cecconi M. Critical care utilization for the COVID-19 outbreak in Lombardy, Italy: early experience and forecast during an emergency response. JAMA 2020;19:2-3.

3. Zhou F, Yu T, Du R, et al. Clinical course and risk factors for mortality of adult inpatients with COVID-19 in Wuhan, China: a retrospective cohort study. Lancet 2020;395:1054-1062.

4. Mao L, Jin H, Wang M, et al. Neurologic manifestations of hospitalized patients with coronavirus disease 2019 in Wuhan, China. JAMA Neurol Epub 2020 Apr 10.

5. Li Y, Wang M, Zhou Y, et al. Acute cerebrovascular disease following COVID-19: a single center, retrospective, observational study. SSRN 2020; Available at SSRN: https://ssrn.com/abstract=3550025.

6. Mao L, Wang M, Chen S, et al. Neurological manifestations of hospitalized patients with COVID-19 in Wuhan, China: a retrospective case series study. JAMA Neurol Epub 2020 April 10.

7. Dato Istat. Popolazione residente al lo gennaio 2019 nella provincia di Brescia. Available at: demo.istat.it/bilmens2019gen/index.html. Accessed April 5, 2020.

8. COVID-19: neurologists in Italy to colleagues in US: look for poorly-defined neurologic conditions in patients with the coronavirus. Available at: journals.lww.com/neurotodayonline/blog/breakingnews/pages/post.aspx?PostID=920. Accessed April 5, 2020

9. Huang C, Wang Y, Li X, et al. Clinical features of patients infected with 2019 novel coronavirus in Wuhan, China. Lancet 2020;395:497-506.

10. Inouye SK, van Dyck $\mathrm{CH}$, Alessi CA, Balkin S, Siegal AP, Horwitz RI. Clarifying confusion: the Confusion Assessment Method: a new method for detection of delirium. Ann Intern Med 1990;113:941-948.

11. Pike N. Using false discovery rates for multiple comparisons in ecology and evolution. Methods Ecol Evol 2011;2:278-282.

12. Seymour CW, Liu VX, Iwashyna TJ, et al. Assessment of clinical criteria for sepsis for the third international consensus definitions for sepsis and septic shock (sepsis-3). JAMA 2016;315:762-774.

13. Guo T, Fan $\mathrm{Y}$, Chen M, et al. Cardiovascular implications of fatal outcomes of patients with coronavirus disease 2019 (COVID-19). JAMA Cardiol Epub 2020 Mar 27.

14. Inciardi RM, Lupi L, Zaccone G, et al. Cardiac involvement in a patient with coronavirus disease 2019 (COVID-19). JAMA Cardiol 2020;2019:4-9.

15. AHA/ASA Stroke Council Leadership. Temporary Emergency Guidance to US Stroke Centers During the Coronavirus Disease 2019 (COVID-19) Pandemic: On Behalf of the American Heart Association/American Stroke Association Stroke Council Leadership. Stroke 2020;51:1910-1912.

16. Davidson JA, Warren-Gash C. Cardiovascular complications of acute respiratory infections: current research and future directions. Expert Rev Anti Infect Ther 2019;17:939-942.

17. Smeeth L, Thomas SL, Hall AJ, Hubbard R, Farrington P, Vallance P. Risk of myocardial infarction and stroke after acute infection or vaccination. N Engl J Med 2004;351:2611-2618.

18. Yan R, Zhang Y, Li Y, Xia L, Guo Y, Zhou Q. Structural basis for the recognition of the SARS-CoV-2 by full-length human ACE2. Science 2020;2:1444-1448.

19. Shang J, Ye G, Shi K, et al. Structural basis of receptor recognition by SARS-CoV-2. Nature 2020;581:221-224.

20. Lan J, Ge J, Yu J, et al. Structure of the SARS-CoV-2 spike receptor-binding domain bound to the ACE2 receptor. Nature 2020;581:215-220.

21. Mendoza Torres E, Oyarzún A, Mondaca RuffD, et al. ACE2 and vasoactive peptides novel players in cardiovascular/renal remodeling and hypertension. Ther Adv Cardiovasc Dis 2015;9:217-237.

22. Hamming I, Timens W, Bulthuis MLC, Lely AT, Navis GJ, van Goor H. Tissue distribution of ACE2 protein, the functional receptor for SARS coronavirus: a first step in understanding SARS pathogenesis. J Pathol 2004;203:631-637.

23. Gallagher PE, Ferrario CM, Tallant EA. Regulation of ACE2 in cardiac myocytes and fibroblasts. Am J Physiol Heart Circ Physiol 2008;295:2373-2379.

24. Levi $\mathrm{M}$, Toh $\mathrm{CH}$, Thachil J, Watson HG. Guidelines for the diagnosis and management of disseminated intravascular coagulation. Br J Haematol 2009; 145:24-33.

25. Tang N, Li D, Wang X, Sun Z. Abnormal coagulation parameters are associated with poor prognosis in patients with novel coronavirus pneumonia. J Thromb Haemost 2020:18:844-847.

26. Grasselli G, Zangrillo A, Zanella A, et al. Baseline characteristics and outcomes of 1591 patients infected with SARS-CoV-2 admitted to ICUs of the Lombardy region, Italy. JAMA 2020:323:1574-1581.

27. Guan WJ, Ni ZY, Hu Y, et al. Clinical characteristics of coronavirus disease 2019 in China. N Engl J Med 2020;19:3-4.

28. Oxley TJ, Mocco J, Majidi S, et al. Large-vessel stroke as a presenting feature of covid19 in the young. N Engl J Med 2020;375:e60.

29. Pilotto A, Odolini Si, Masciocchi S, et al. Steroid-responsive severe encephalopathy in SARS-CoV-2 infection. Ann Neurol Epub 2020 May 17.

30. Dinkin M, Gao V, Kahan J, et al. COVID-19 presenting with ophthalmoparesis from cranial nerve palsy. Neurology Epub 2020 May 1.

31. Alberti P, Beretta S, Piatti M, et al. Guillain-Barré syndrome related to COVID-19 infection. Neurol Neuroimmunol Neuroinflammation 2020;7:1-4.

32. Gutiérrez-Ortiz C, Méndez A, Rodrigo-Rey S, et al. Miller Fisher syndrome and polyneuritis cranialis in COVID-19. Neurology Epub 2020 Apr 17.

33. White $\mathrm{DB}, \mathrm{Lo} \mathrm{B}$. A framework for rationing ventilators and critical care beds during the COVID-19 pandemic. JAMA 2020;146:e61S-e74S

34. Cohen IG, Crespo AM, White DB. Potential legal liability for withdrawing or withholding ventilators during COVID-19: assessing the risks and identifying needed reforms. JAMA Epub 2020 Apr 1. 


\section{Neurology}

\section{Clinical characteristics and outcomes of inpatients with neurologic disease and COVID-19 in Brescia, Lombardy, Italy}

Alberto Benussi, Andrea Pilotto, Enrico Premi, et al.

Neurology 2020;95;e910-e920 Published Online before print May 22, 2020

DOI 10.1212/WNL.0000000000009848

This information is current as of May 22, 2020

Updated Information \&
Services
References
Citations
Subspecialty Collections
Permissions \& Licensing
Reprints

\section{Updated Information \&}

References

Citations

\section{Subspecialty Collections}

Reprints including high resolution figures, can be found at: http://n.neurology.org/content/95/7/e910.full

This article cites 22 articles, 0 of which you can access for free at: http://n.neurology.org/content/95/7/e910.full\#ref-list-1

This article has been cited by 3 HighWire-hosted articles: http://n.neurology.org/content/95/7/e910.full\#\#otherarticles

This article, along with others on similar topics, appears in the following collection(s):

\section{All Cerebrovascular disease/Stroke}

http://n.neurology.org/cgi/collection/all_cerebrovascular_disease_strok $\mathrm{e}$

Prognosis

http://n.neurology.org/cgi/collection/prognosis

Information about reproducing this article in parts (figures,tables) or in its entirety can be found online at:

http://www.neurology.org/about/about_the_journal\#permissions

Information about ordering reprints can be found online:

http://n.neurology.org/subscribers/advertise

Neurology ${ }^{\circledR}$ is the official journal of the American Academy of Neurology. Published continuously since 1951, it is now a weekly with 48 issues per year. Copyright () 2020 American Academy of Neurology. All rights reserved. Print ISSN: 0028-3878. Online ISSN: 1526-632X.

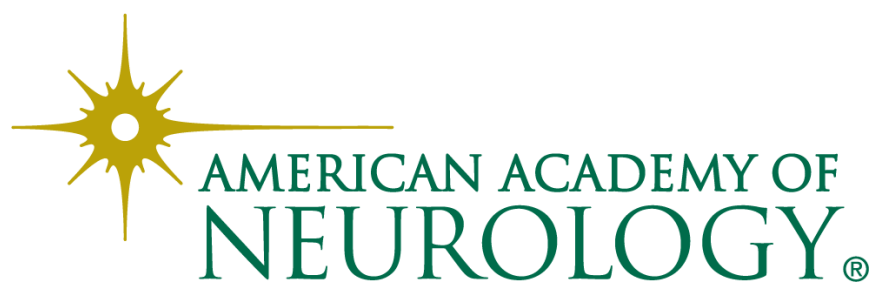

